\title{
Glyceraldehyde-3-Phosphate Dehydrogenase: A Promising Target for Molecular Therapy in Hepatocellular Carcinoma
}

\author{
Shanmugasundaram Ganapathy-Kanniappan'1, Rani Kunjithapatham¹, Jean- \\ Francois Geschwind ${ }^{1}$ \\ ${ }^{1}$ Department of Radiology \& Radiological sciences, Johns Hopkins University School of Medicine, Baltimore, MD, USA \\ Correspondence to: Jean-Francois Geschwind, email: jfg@jhmi.edu \\ Keywords: 3-bromopyruvate, GAPDH, Glycolysis, HCC, lodoacetate, Koningic acid, Methylglyoxal, Saframycin A \\ Received: August 15, 2012, Accepted: September 06, 2012, Published: September 07, 2012
}

Copyright: () Ganapathy-Kanniappan et al. This is an open-access article distributed under the terms of the Creative Commons Attribution License, which permits unrestricted use, distribution, and reproduction in any medium, provided the original author and source are credited.

\section{ABSTRACT:}

Hepatocellular carcinoma (HCC) is one of the most highly lethal malignancies ranking as the third leading-cause of cancer-related death worldwide. Although surgical resection and transplantation are effective curative therapies, very few patients qualify for such treatments due to the advanced stage of the disease at diagnosis. In this context, loco-regional therapies provide a viable therapeutic alternative with minimal systemic toxicity. However, as chemoresistance and tumor recurrence negatively impact the success of therapy resulting in poorer patient outcomes it is imperative to identify new molecular target(s) in cancer cells that could be effectively targeted by novel agents. Recent research has demonstrated that proliferation in HCC is associated with increased glucose metabolism. The glycolytic enzyme, glyceraldehyde-3-phosphate dehydrogenase (GAPDH), a multifunctional protein primarily recognized for its role in glucose metabolism, has already been shown to affect the proliferative potential of cancer cells. In human HCC, the increased expression of GAPDH is invariably associated with enhanced glycolytic capacity facilitating tumor progression. Though it is not yet known whether GAPDH up-regulation contributes to tumorigenesis sensu stricto, emerging evidence points to the existence of a link between GAPDH up-regulation and the promotion of survival mechanisms in cancer cells as well as chemoresistance. The involvement of GAPDH in several hepatocarcinogenic mechanisms (e.g. viral hepatitis, metabolic alterations) and its sensitivity to a new class of prospective anticancer agents prompted us to review the current understanding of the therapeutic potential of targeting GAPDH in HCC.

Glyceraldehyde-3-phosphate dehydrogenase (GAPDH) is a classical protein with enormous biochemical and biophysical interests owing to its functional significance in glucose metabolism. The focus on GAPDH has gained further momentum with recent discoveries unraveling its non-glycolytic (nonenzymatic) roles associated with cell death and diseases. Pharmacologically, GAPDH obeys the cardinal rules of "druggability" due to its disease relevance and the presence of an inhibitory site (e.g. catalytic domain of an enzyme, ligand-binding site of a receptor). Although, it has been estimated that at least $2-5 \%$ of the human genome (among $\sim 30,000$ genes) may form potential therapeutic targets [1], the key requirements of "druggability", in the interests of pharmaceutical industry or any drugdevelopment programs, restrict the number dramatically into fewer candidates. Nonetheless, the efficacy of the agent(s) targeting such a "druggable molecule" and the tolerable systemic toxicity and so on, will eventually determine the fate of successful clinical translation. Current advancement in understanding the cancerrelated roles of GAPDH together with the sensitivity of tumor cells to its inhibition designates GAPDH as a potential molecular target in cancer treatment. Several elegant reviews by Sirover and others have delineated the functional diversity of GAPDH, particularly its participation in cell death and survival mechanisms [2-6]. The aim of the current review is to critically evaluate, from 
an interventional oncology perspective, the druggability of GAPDH and its therapeutic potential in liver cancer.

\section{Liver cancer and current therapeutic challenges}

Liver cancer is one of the most lethal malignancies and consistently ranked as the third most common cause of cancer-related death worldwide [7]. Intense research both at preclinical and clinical levels contributed a wealth of information on the causes and risk factors of liver cancer. In general, functional impairment of normal liver by hepatitis (due to the infection of hepatitis $B$ and $C$ viruses), excessive alcohol consumption, and aflatoxin $\mathrm{B}$, in addition to oncogenic driver mutations, are considered as some of the causal factors that promote cirrhosis eventually leading to liver cancer [e.g. hepatocellular carcinoma (HCC)] (Figure 1). Statistically, the incidence of HCC in the Unites States is on the rise, secondary to a parallel rise in hepatitis $\mathrm{C}$ virus ( $\mathrm{HCV})$. In $\mathrm{HCC}$, patient survival remains poor at less than nine months, and largely depends on the stage of the disease. Patients with intermediate-stage disease show better 3-year survival expectancy than advanced-stage disease (viz., 50\% vs. 8\%) [8]. Current curative therapies include surgical resection and transplantation, and are very effective in early-stage disease, however very few patients qualify for such treatments. The asymptomatic nature of HCC and the lack of an early-detection marker invariably result in the diagnosis of the disease at an advanced stage enforcing the patients to other treatment modalities. Among other therapeutic options for HCC, loco-regional therapies have the unique advantage of selective-targeting of tumors (primarily under image guidance), thereby evading systemic toxicity [9]. In clinics, the loco-regional therapies in practice consist of intra-arterial chemo-embolization or radio-embolization $[10,11]$ and percutaneous ablative therapies [12-15]. The effectiveness of locoregional targeted-delivery in achieving tumor ablation greatly impacted the development of new, potential chemotherapeutic agents of high target-specificity and tolerable-toxicity profile. Nevertheless, as HCC is always associated with an underlying disease, it is imperative to administer any novel agent that selectively targets tumor cells with a high level molecular specificity. Recently, there has been a tremendous progress in the development of various therapeutics targeting different pathways or molecules of $\mathrm{HCC}[16,17]$, yet the therapeutic success is often counteracted by chemoresistance and tumor recurrence necessitating the search for sensitive target molecule(s) that can be effectively targeted by potent inhibitors/drugs.

\section{Rationale for molecular targeting of tumor metabolism in liver cancer}

Liver cancer has long been known to have increased-glucose metabolism, a prominent biochemicalsignature of solid tumors. This tumor-specific metabolic

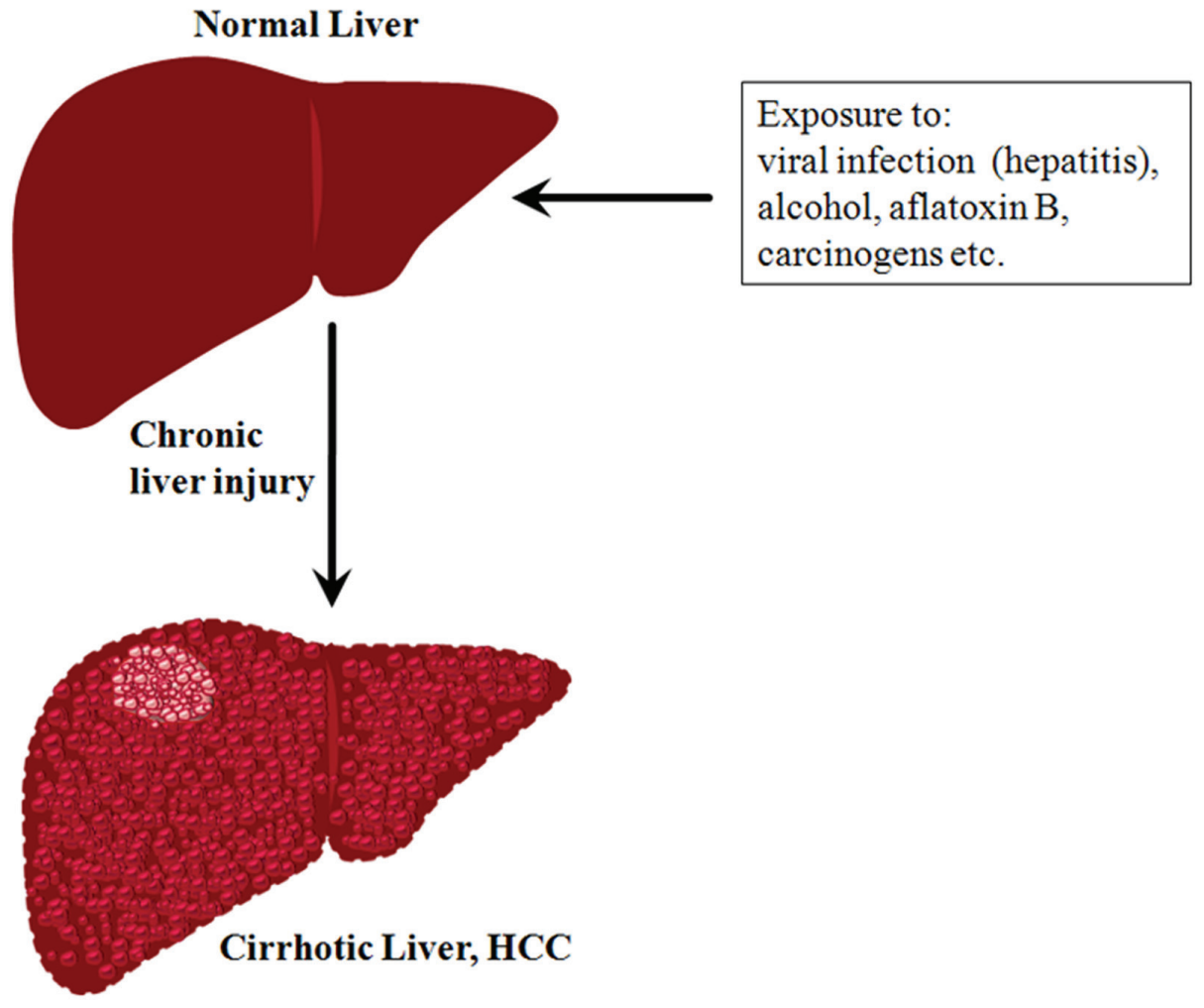

Figure 1: A schematic representation showing the transformation of normal liver into cirrhotic liver leading to HCC. 
phenotype plays pivotal roles in several biosynthetic processes facilitating uninterrupted growth. Further, recent data indicate that oncogenic driver mutations culminate in altered signal transduction pathways enabling tumor cells to reprogram their metabolic circuitry to adapt to the microenvironment. For example, it has been demonstrated that enhanced nutrient uptake is an effect of oncogenic $R A S$ mutations [18]. Similarly, the tumor suppressor, p53, which has been known to be mutated in majority of tumors, has a role in the regulation of glucose metabolism [19]. Mounting evidence indicates that the tumorspecific shift in metabolism is vital for the uncontrolled proliferation and invasiveness of almost all solid tumors. Alternatively, this disparity in glucose metabolism between tumor cells and normal cells suggests a window of opportunity in treating cancer. This altered metabolism has been exploited by PET imaging in clinical diagnosis in the detection of malignant tumors using the glucose analog, fluorodeoxyglucose (FDG). Thus, tumor (glucose) metabolism has been recognized as critical for tumor growth, hence aptly described as "Cancer's Achilles' Heel" [20] accentuating it as a potential therapeutic target $[21,22]$. Hence, the ramifications of disrupting glucose metabolism could be envisaged to generate desirable anticancer effects.

Strong data provide the scientific rational for targeting glucose metabolism in treating $\mathrm{HCC}$, or liver cancer in general. HCC has long been known to demonstrate regulation of glycolytic enzymes facilitating aerobic glycolysis [23]. It has also been demonstrated that in HCC, tumor proliferation is tightly correlated with glucose metabolism [24]. Indeed, both increased-glucose metabolism and proliferation share common regulatory pathways, making tumor metabolism a unique therapeutic target [25]. The therapeutic potential of targeting tumor metabolism triggered a renewed interest in studying glucose-metabolism. Consequently our understanding of the molecular regulation of tumor glycolysis has advanced significantly [26]. Many new investigational agents with potential inhibitory effects on glycolysis have been developed, and evaluated both in vitro and in vivo models. Despite promising results in preclinical tumor models [27], the majority of these agents have not been successfully translated into the clinic thus far, either due to the lack of efficacy in the clinical setting and/ or significant systemic toxicity. Thus, the need to identify a molecular target that

Table 1: GAPDH antagonists in preclinical investigations

\begin{tabular}{|c|c|c|}
\hline Antagonist & Key Reference(s) & Experimental Evidence \\
\hline Iodoacetate & McKee et al. 1965 & $\begin{array}{l}\text { In vitro evidence for partial inhibition of GAPDH by } \\
\text { iodoacetate }\end{array}$ \\
\hline Koningic acid & $\begin{array}{l}\text { Endo et al., } 1985 \\
\text { Kumagai et al., } 2008\end{array}$ & $\begin{array}{l}\text { In vitro evidence documenting the inhibition of GAPDH } \\
\text { Ehrlich ascites suppression by GAPDH inhibition in vivo }\end{array}$ \\
\hline $\begin{array}{l}\text { 3-Bromopyruvate } \\
\text { (3-BrPA) }\end{array}$ & $\begin{array}{l}\text { Barnard et al., } 1993 \\
\text { Geschwind et al., } 2002 \\
\text { Pereira de Silva et al., } 2009 \\
\text { Ganapathy-Kanniappan } \\
\text { et al., (2009) } \\
\text { Ganapathy-Kanniappan } \\
\text { et al., (2012) }\end{array}$ & $\begin{array}{l}\text { First report indicating 3-BrPA binding with GAPDH } \\
\text { First loco-regional therapy demonstrating anticancer } \\
\text { efficacy of 3-BrPA } \\
\text { First biochemical evidence in human HCC cells indicating } \\
\text { that GAPDH and could be the primary target of 3-BrPA } \\
\text { First autoradiographic evidence in human HCC cells } \\
\text { demonstrating GAPDH as the primary target of 3-BrPA } \\
\text { First report demonstrating percutaneous ablation of human } \\
\text { HCC by targeting GAPDH through 3-BrPA }\end{array}$ \\
\hline Methylglyoxal & $\begin{array}{l}\text { Ray et al., } 1997 \\
\text { Lee et al., } 2005\end{array}$ & $\begin{array}{l}\text { Demonstrates GAPDH of tumor cells as the principal target } \\
\text { In vitro evidence showing biochemical modification of } \\
\text { GAPDH by methylglyoxal }\end{array}$ \\
\hline Saframycin A & Xing et al., 2004 & $\begin{array}{l}\text { In vitro evidence demonstrating GAPDH as the target of } \\
\text { Saframycin A -DNA adducts. Suggests GAPDH could be a } \\
\text { chemotherapeutic target }\end{array}$ \\
\hline Oligonucleotide & Kim et al., 1999 & $\begin{array}{l}\text { First report showing that GAPDH inhibition by antisense } \\
\text { oligonucleotides affects proliferation, and induces apoptosis } \\
\text { in cancer cells }\end{array}$ \\
\hline $\begin{array}{l}\text { siRNA } \\
\text { shRNA }\end{array}$ & $\begin{array}{l}\text { Phadke et al., (2009) } \\
\text { Ganapathy-Kanniappan } \\
\text { et al., (2012) }\end{array}$ & $\begin{array}{l}\text { Demonstrates that GAPDH-siRNA induces cell cycle arrest } \\
\text { First report demonstrating antitumorigenic effects of } \\
\text { GAPDH silencing in human HCC both in vitro and in vivo }\end{array}$ \\
\hline
\end{tabular}


is indispensable for cancer cell survival and developing an agent to effectively inhibit the molecule remains critical for successful anticancer therapy.

\section{GAPDH in hepatocarcinogenic mechanisms}

Several reports unravel the participation of GAPDH in pathways that are cross-linked with cancer-specific or cancer-related phenotypes. GAPDH has been known to interact with the nucleic acids of Hepatitis B [28, 29] and $\mathrm{C}[30]$ viruses that cause hepatitis, a major contributing factor for hepatocarcinogenesis. Although GAPDH binding with nucleic acids of other viruses have also been reported (e.g. influenza virus, Japanese encephalitis virus) $[31,32]$, none of those viruses have been significantly associated with hepatocarcinogenesis or any other carcinogenesis.

Accumulating data indicate a strong link between GAPDH up-regulation and tumorigenic potential of transforming cells. For example, granulocyte macrophage (GM) colony-stimulating factor-1 (CSF-1), a factor known to play a pivotal role in several malignancies, has been increasingly recognized to be associated with hepatocarcinogenesis [33-35]. Surprisingly, GAPDH has been known to bind with CSF-1 mRNA resulting in increased stability, thus contributing for tumorigenic potential or malignant phenotype [36]. Moreover, analysis of HCC patient samples revealed the incidence of GAPDH up-regulation in human HCC strongly correlates with $c$-jun, a proto-oncogene that has long been known to be involved in liver tumorigenesis [37]. It remains to be seen if such a correlation exists between GAPDH expression and other oncogenes (e.g. KRAS) in HCC progression. Though it is not known whether GAPDH up-regulation contributes for tumorigenesis sensu stricto, adequate data unequivocally demonstrate the existence of an association between GAPDH over-expression and pro-survival mechanisms [5] and chemoresistance [38] in cancer cells.

Metabolically, in human HCC, the increased expression of GAPDH is invariably associated with increased glycolytic capacity [23, 39], facilitating tumor progression (Figure 2). Recently, a hitherto unknown role of GAPDH in the regulation of mammalian target of rapamycin (mTOR)-complex1 (mTOR-C1) signaling pathway has been documented [40]. mTOR pathway is a growth signaling mechanism that has been active during hepatocarcinogenesis [41, 42], hence provides

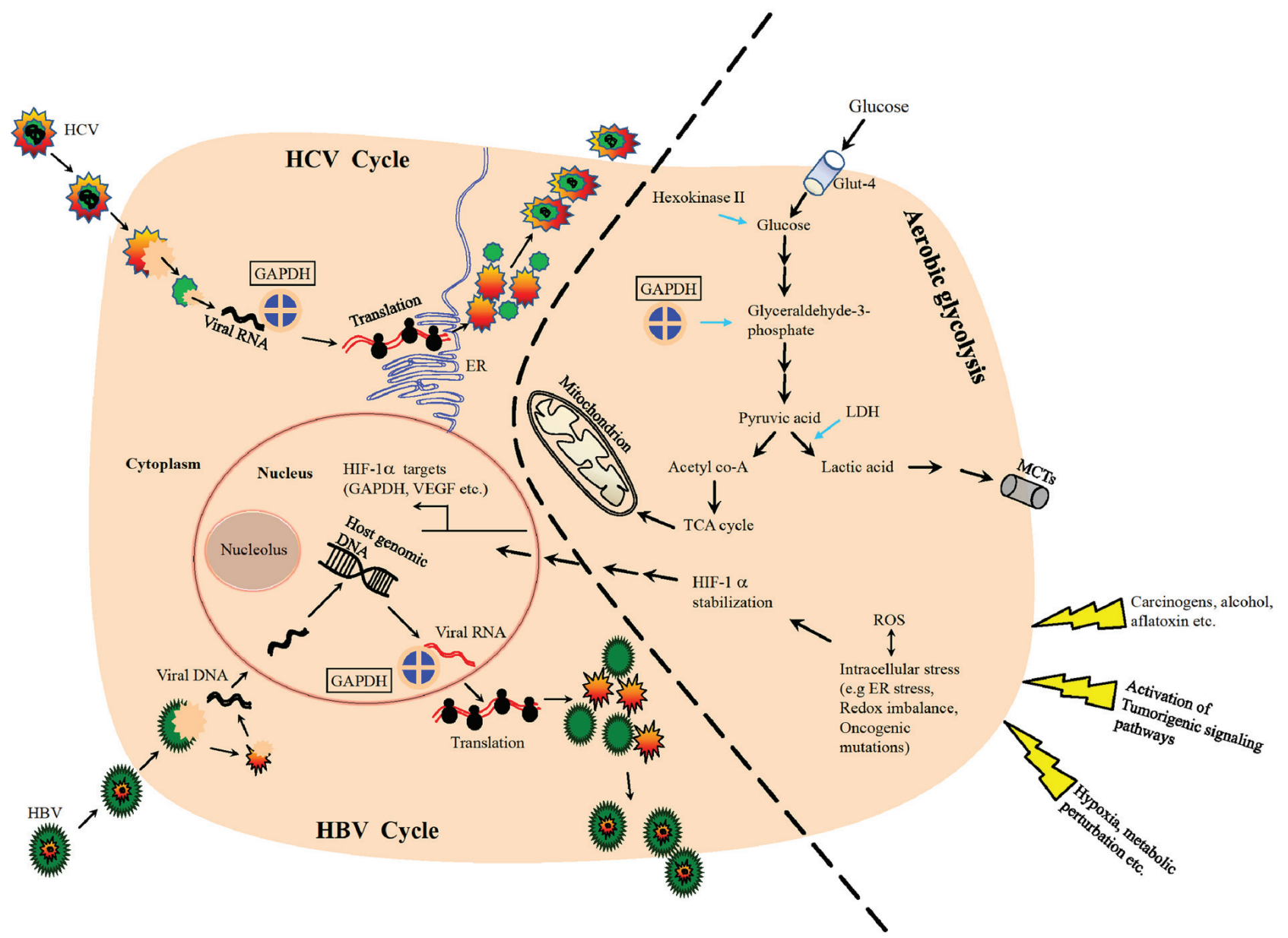

Figure 2: A schematic showing the involvement of GAPDH in hepatocarcinogenic mechanisms. 
an opportunity for therapeutic targeting [43-48]. It has been found that GAPDH regulates mTOR-C1 signaling according to the availability of glucose (hence ATP). GAPDH interaction with rheb, the activator of mTOR, mitigates mTOR-C1 signaling pathway thus halting the growth mechanism under conditions of energy depletion or inadequate glycolysis. It has to be noted that only chronic disruption but not transient inhibition of mTOR affects tumor cells [49]. Thus, GAPDH in addition to its role in glycolysis and energy production also signals and regulates other pathways like mTOR, depending upon cellular requirements. Hence, GAPDH is one of the very few earliest metabolic enzymes to exert such pleiotropic effects on energy metabolism and related signaling pathways. Unlike the majority of metabolic enzymes, GAPDH displays the unique feature of being functionally active in all three cellular compartments; nucleus, cytoplasm and plasma membrane. As a result, the interaction of GAPDH with either proteins or nucleic acids has been attributed to its diverse functions. Such interactions have been known to result in a variety of effects; (a) its interaction with VDAC-1 initiating proapoptotic membrane permeabilization of mitochondria
[50], (b) its binding with tubulin affecting cytoskeletal structures and membrane trafficking and (c) its localization within endoplasmic reticulum (ER) affecting secretory pathway. GAPDH also interacts with RNA (e.g. human tRNA, lymphokine mRNA), and DNA (e.g. binding with telomere $[38,51])$, and has been known to impact the genetic regulation of Oct-1 by participating in the OCA-s complex.

Such a multifaceted role for a single protein (GAPDH) would nevertheless require complex structural organization and regulation under dynamic physiological conditions. In this context the role of posttranslational modification (PTM) of GAPDH has been critically analyzed. It has been well documented that GAPDH undergoes one or more modifications such as acetylation, O-GlcNAcylation, S-nitrosylation, thiolation and "siah-1binding" depending upon its cellular function. All of these PTMs have already been known to affect the enzymatic function of GAPDH [52]. In other words, PTMs that underlie GAPDH's non-glycolytic roles eventually affects its glycolytic function.

Since it has been established that GAPDH achieves its non-glycolytic functions through PTMs, compromising

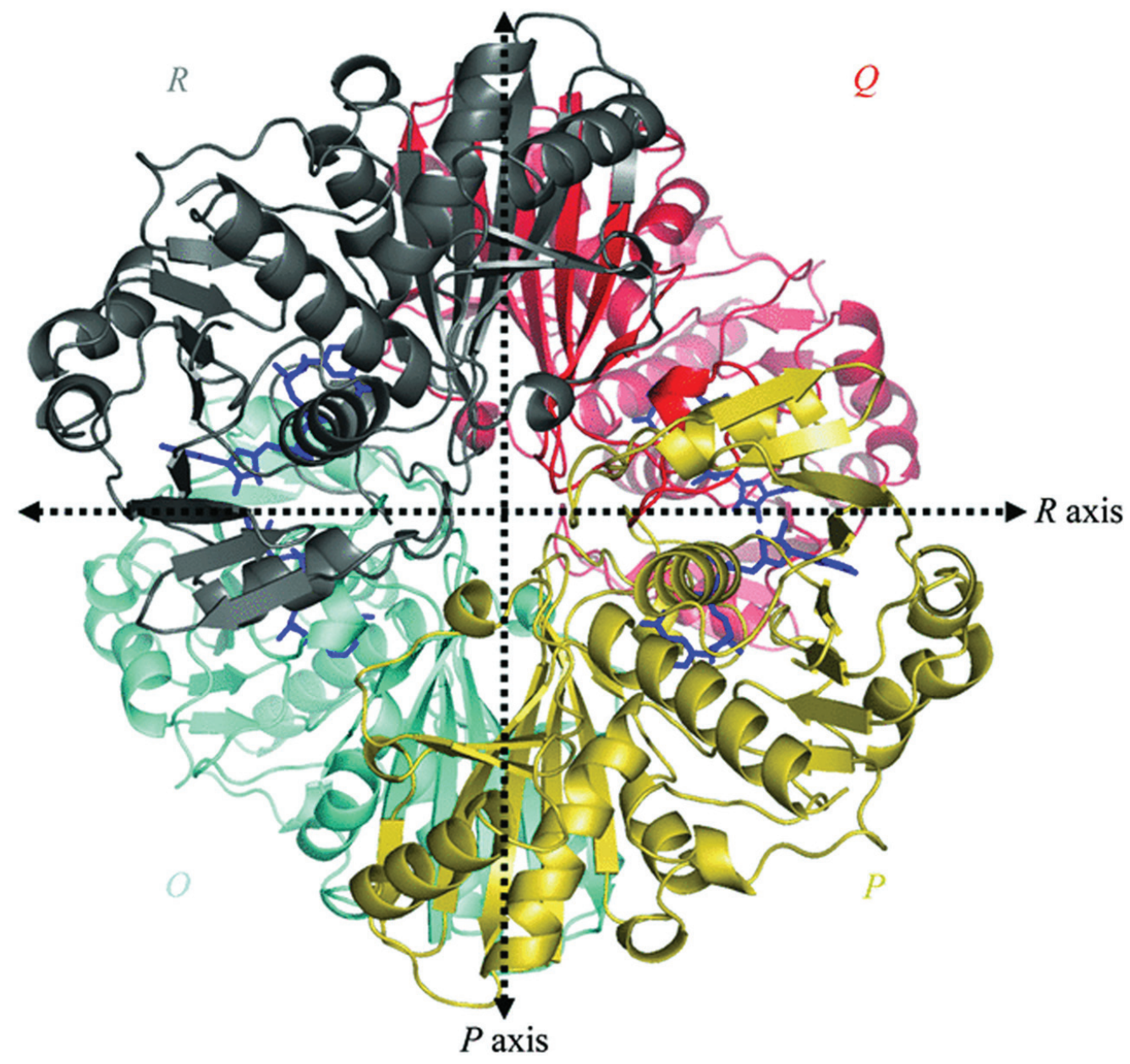

Figure 3: Overall view of the homotetramer of human liver GAPDH [44] (Reproduced with permission from http:// dx.doi.org/10.1107/S0907444905026740). 
its glycolytic role conceivably, it would have serious consequences on the bioenergetics of cancer cells. Nevertheless, its cellular abundance and cancer-specific over-expression could compensate or satisfy for the variety of functions while maintaining tumor glycolysis. From an experimental therapeutic point of view, the multiple roles of GAPDH such as enzymic function (in energy metabolism), scaffold or adaptor protein (in vesicular trafficking) makes GAPDH an attractive molecular target to achieve desired anticancer effects. In other words, blockade of GAPDH would impair multiple pathways / mechanisms forcing cancer cells to become fragile, eventually causing their death.

\section{Targeting GAPDH to treat liver cancer}

Considering a therapeutic strategy to target GAPDH primarily relies on (a) its disease-relevance and (b) druggability. The rationale for considering GAPDH as a molecular target, from an oncologist's perspective, chiefly depends on its disease-relevance and the desirable phenotypic effects of abrogating its functions. Whereas, for a pharmacologist, the accessibility and inhibition of various domains of GAPDH by developing specific inhibitors to block its biochemical functions provides the rationale for drug design (Figure 3). GAPDH enzyme exists as a tetramer (four subunits) with each subunit having a catalytic site favoring the inhibition of the native enzyme even at any one of the four catalytic sites to disrupt its function.

Though enzymes such as lactate dehydrogenase (LDH) [53] and hexokinase II (HKII) [54] have been investigated in preclinical tumor models for their therapeutic potential, their respective inhibitors are yet to be successful in clinical trials. Hence, the need to identify a target that is critical for tumor growth but sensitive to therapy remains unanswered. As discussed elsewhere, the tumor specific roles of GAPDH include, apart from its glycolytic function, chemoresistance [38, 55], metastatic potential [56], protecting cells against caspaseindependent apoptosis [5] and cell cycle regulation [57]. Thus, any interference with GAPDH's function is anticipated to surpass the effect of a single molecule (e.g. LDH, HK II) targeted-therapy that exclusively targets one molecule, and one pathway/function, where the interrupted physiology could be compensated by collateral or alternative mechanisms. Conceivably, GAPDH inhibition could sensitize the cancer cells for chemotherapy, as the protection and resistance offered by GAPDH will also be abrogated. However, the concern of ubiquitous nature of GAPDH, and the related systemic toxicity, needs to be addressed.

In this context, advancements in current interventional approaches (like loco-regional therapies, thermal-, cryo-, and chemo-ablative therapies, nanoparticle or lysosome-based drug delivery etc. ) and identification of GAPDH-specific inhibitor(s) provide impetus in exploring the therapeutic potential associated with the anti-GAPDH<smiles>COC1=C(C)C(=O)C2=C(C1=O)[C@@H](CNC(=O)C(C)=O)N1[C@@H]3C4=C(C[C@H]1[C@H](C#N)C[C@@H]3C2=O)C(=O)C(C)=C(OC)C4=O</smiles>

Koningic acid $\left(\mathrm{C}_{15} \mathrm{H}_{20} \mathrm{O}_{5}\right)$

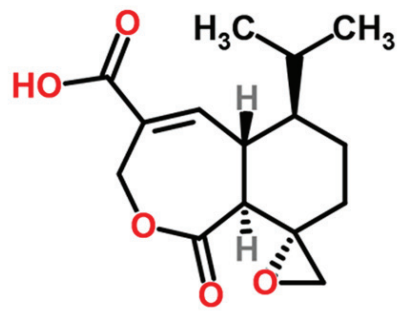

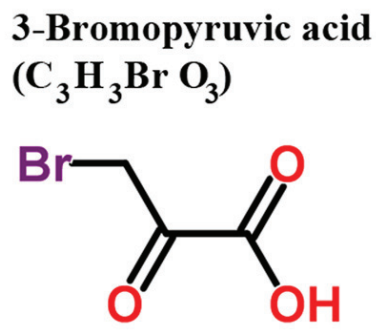
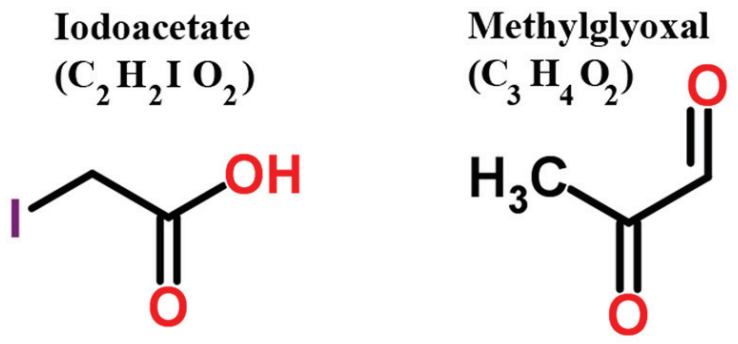

Figure 4: Structure of various inhibitors of GAPDH with anticancer effects in preclinical models (Reproduced with permission of RSC Worldwide Ltd from http://www.chemspider.com). 
therapeutic strategy. Thus, molecular targeting of GAPDH by tumor-specific delivery or inhibition could provide a viable therapeutic opportunity enabling us to overcome the current challenges in chemotherapy.

\section{Preclinical efficacy of GAPDH inhibitors}

Several inhibitors derived from natural and / or synthetic sources have been investigated for their antiGAPDH efficacy in treating cancer (Figure 4, Table I). Here, we discuss the agents that have been investigated in preclinical models that demonstrate adequate data on anticancer efficacy of such inhibitors.

\section{Saframycin (Saf A)}

Myers and his co-workers [58] elegantly demonstrated the involvement of GAPDH in the antiproliferative effects of Saframycin A (Saf A), a natural product of bacterial fermentation. This report suggested GAPDH as a protein target of chemotherapeutic agents. However, the principal mechanism underlying the antiproliferative effects of Saf A involves the formation of DNA adducts as the first step followed by the binding of the drug-DNA adduct with GAPDH protein. Although GAPDH is implicated in the anti-proliferative effects mediated by Saf A class agents, additional experimental evidence is required to demonstrate if Saf $\mathrm{A}$ is a specific inhibitor of GAPDH.

\section{Koningic acid (KA)}

The fungal metabolite Koningic acid (KA) has been reported to inhibit glycolysis through the inhibition of GAPDH $[59,60]$. The antiglycolytic principle of KA has been documented in multiple in vitro models. The efficacy of KA has been shown to be directly proportional to the glycolytic- dependency of cells, with cells exhibiting increased glycolysis demonstrating higher sensitivity [61]. KA binding site with GAPDH and the subsequent inhibition of activity has been identified. Preliminary reports have also demonstrated that administration of KA within 8 days of intraperitoneal inoculation of Ehrlich ascites tumor cells provided survival benefit to mice compared to untreated placebo, although detailed reports on the effect of KA on the rate of tumor growth and other tumor types are wanting. Further investigations on KA with a focus on selective targeting of tumor-GAPDH and not normal cellular GAPDH would provide an opportunity in understanding and advancing its therapeutic potential.

\section{3-Bromopyruvate (3-BrPA)}

The metabolic blocker, 3-bromopyruvate (3BrPA), a halogenated analog of pyruvic acid, has gained significant attention due to its remarkable antitumor effects. In vitro testing against human $\mathrm{HCC}$ cells demonstrated that 3-BrPA inhibited glycolysis and blocked ATP production causing apoptosis in a dosedependent manner [62]. Tracer studies with radio- $\left({ }^{14} \mathrm{C}\right)-$ labeled 3-BrPA demonstrated GAPDH as the primary intracellular target of 3-BrPA. The binding of 3-BrPA

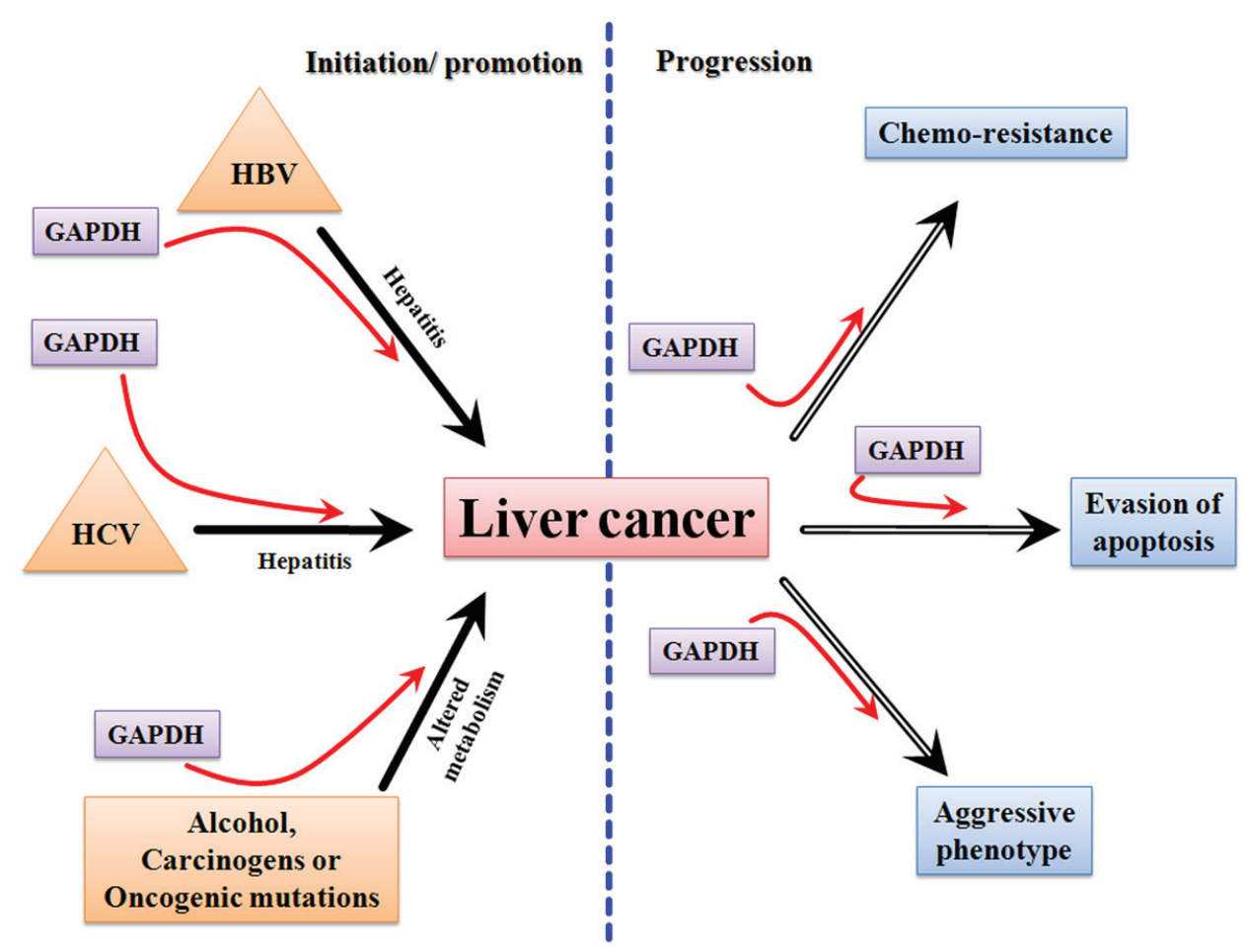

Figure 5: A schematic diagram showing the involvement of GAPDH in the processes related to the initiation/promotion and progression of hepatocarcinogenesis. 
to GAPDH in multiple cell lines [63, 64] substantiated the fact that GAPDH inhibition underlies 3-BrPA's antiglycolytic effect, leading to apoptotic cell death [64]. 3 -BrPA is one of the very few agents that have substantial data demonstrating GAPDH as the preferred target. Unlike other alkylating agents, 3-BrPA demonstrated tremendous specificity in molecular targeting, enforcing its antitumorigenic effects by promoting energy depletion, disruption of redox balance and induction of intracellular stress in a concurrent fashion. Therefore it appears that $3-\mathrm{BrPA}$ is an extremely promising agent due to its tumor selectivity and ability to promote a multipronged antitumor effect enabling it to progress towards clinical trials.

\section{Iodoacetate (IA)}

McKee et al., [65] provided the earliest documentation of Iodoacetate (IA)'s inhibitory role on GAPDH in cancer cells, where the effect of GAPDH inhibition was seen in micromolar concentrations of IA. Interestingly at the lowest concentration (3 micromoles) used by these investigators the cancer cells switched from glycolysis to oxidative phosphorylation (as evident by an increase in oxygen consumption and a decrease in lactate production) with no signs of cytotoxicity. However, currently IA's use as a metabolic blocker is at higher concentrations ( $\sim 100$ micromoles) where significant cytotoxicity occurs [66]. Although the metabolic inhibitory effect of IA has been attributed to its effect on GAPDH activity, further investigations are required to validate GAPDH as the primary target of IA.

\section{Methylglyoxal (MG)}

Methylglyoxal (MG) a normal metabolite of glucose metabolism is formed during the process of glycolysis by dephosphorylation of glyceraldehyde-3-phosphate or dihydroxyacetone [67]. Methylglyoxal has been reported to inhibit tumorigenesis by interfering with the growth of malignant cells [68]. In the past decade reports have indicated that methylglyoxal targets the enzyme GAPDH, and this inhibition is the primary mechanism underlying its anticancer effects [69]. Lee et al., [67] have also demonstrated that the physical interaction / binding of methylglyoxal resulted in an alteration of the structure of GAPDH leading to inactivation. Methylglyoxal mediated glycation has been attributed as the cause for such an inactivation of GAPDH. Although very interesting, additional experimental evidence validating the tumor selectivity and molecular specificity (showing GAPDH as the only or at least the primary target) of MG in multiple

A

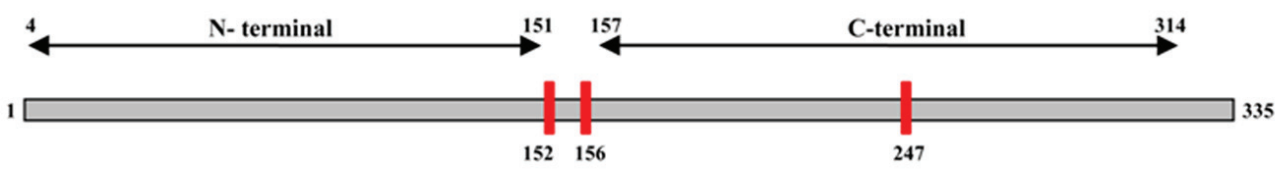

B

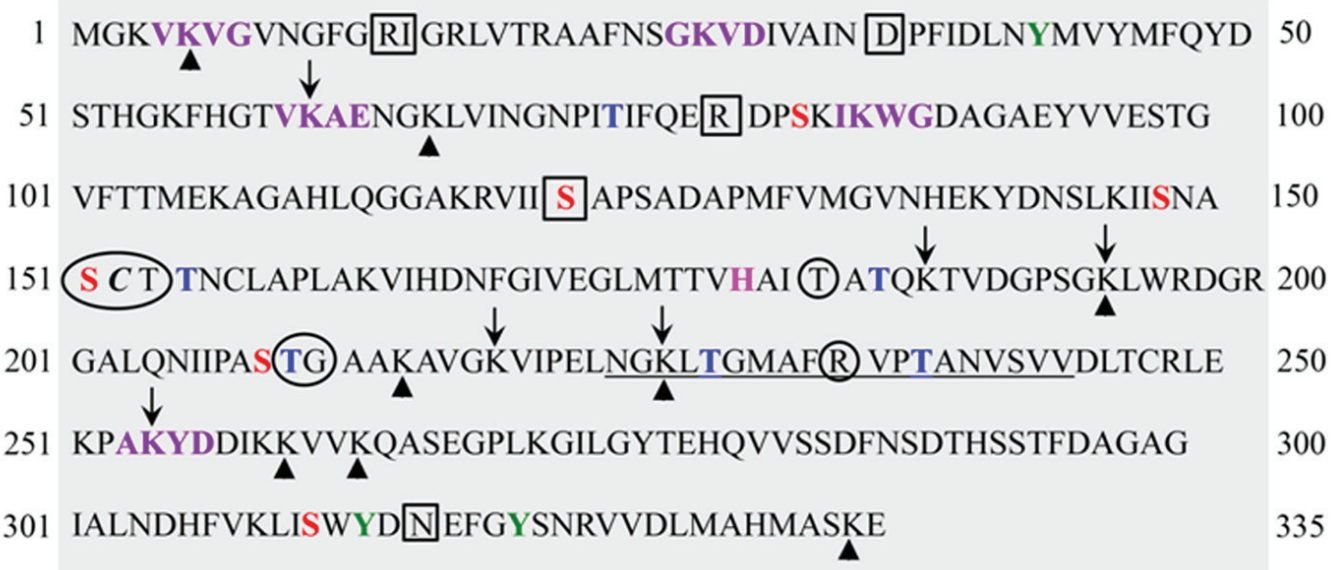

Figure 6: Human liver GAPDH showing various motifs. (A). Line diagram showing the N-terminal and C-terminal regions with Cysteine residues (red bars). The amino acid residues numbered as 152 to 156 correspond to the catalytic site. Note: two cysteine residues located in the catalytic site are sensitive targets for majority of the GAPDH inhibitors.

(B). The peptide sequence of GAPDH subunit indicating various posttranslational modification sites. Circles (O) represent Glyceraldehyde-3 phosphate binding sites; Square Boxes $(\llcorner)$ represent NAD binding sites; Red font represents phosphoserine sites; Blue font represents phosphothreonine sites; Pink font represents aminoacid residue that Activates thiol group during catalysis; Green font represents phosphotyrosine sites; Purple font represents Predicted Sumoylation sites (70-90\% probability); Italic font represents S-nitrosylation site and Underlined font represents SIAH-1 binding site. Arrow-head (4) indicates predicted methylation sites while the with Down-ward Arrow $(\downarrow)$ indicates acetylation sites. Region 2-148 is the domain involved in the interaction WARS (tryptophan-tRNA ligase). 
tumor models will help further advancement of MG.

\section{RNAi}

Several reports indicate that silencing GAPDH by antisense oligonucleotides [70] or small-interfering (si) RNA [57] induces apoptosis or affects cell proliferation, in vitro. To our knowledge, until our recent documentation [71], there was no report on the plausibility of shRNAmediated GAPDH knockdown in the management of HCC. Yet, two major challenges to this approach remain. They are; (i) the ubiquitous nature of GAPDH, raising the concern for non-specific toxicity and (ii) its intracellular abundance, which would require higher doses of drug for therapeutic effect. To overcome these challenges, loco-regional therapies such as percutaneous and intra-arterial deliveries provide a viable alternative to systemic administration $[72,73]$. The unique advantage of selectively targeting tumors under image guidance would minimize systemic toxicity [9]. Data demonstrated that percutaneous injection of GAPDH-antagonists block HCC progression. Future studies on the optimization of treatment regimen as well as delivery system with GAPDH antagonists will enable us to characterize the means of achieving maximal therapeutic efficacy either alone or in combination with other therapies. For instance, nanoparticle based slow release of potential therapeutic agents has emerged as a promising approach in preclinical models [74, 75]. Hence, integration of GAPDH antagonists with nanoparticles will allow us to improve the efficacy of therapy with minimal number of percutaneous injections, which is feasible in clinical translation. In summary, molecular targeting of GAPDH via percutaneous injection of either an inhibitor, 3-BrPA, or shRNA blocks tumor progression demonstrating the therapeutic potential of targeting GAPDH in HCC.

\section{Selective targeting of GAPDH}

The involvement of GAPDH in several mechanisms that are associated with hepatocarcinogenesis (e.g. viral hepatitis, metabolic alterations), and the sensitivity of human HCC to targeted inhibition of GAPDH underscore the therapeutic potential of inactivating GAPDH, particularly in liver cancer. Next, the efficacy of targeted inhibition and the necessity of preventing systemic toxicity are the tasks to be achieved for clinical translation of such anti-GAPDH approach.

\section{Loco-regional therapies}

Image-guided procedures, especially intra-arterial therapies, play a key role in the treatment of patients with liver cancer $[10,76]$. The advantage of loco-regional approaches is that it provides not only access to the core but also to the periphery of the tumor. In addition, much greater drug concentrations can be achieved within tumors while minimizing systemic exposure. For example, the intra-arterial delivery of 3-BrPA in animal models of liver cancer demonstrated striking antitumor effects $[77,78]$ in most cases significantly prolonged the survival $[71,79]$. In addition, combinatorial approaches can also be envisaged for improved or better effects in cases where such dual therapies are required. Recently, a clinical investigation revealed a marked effect of combining sorafenib with drug-eluting beads where the patient response was significant [80], assuring the feasibility and effectiveness of such loco-regional therapies.

\section{Tumor specific silencing of GAPDH}

The therapeutic potential of RNA interference (RNAi) strategy for treating $\mathrm{HCC}$ has been increasingly recognized [81]. Despite attempts by several groups, the only RNAi therapeutic against liver tumor that has entered Phase I trial is ALN-VSP02 that targets kinesinspindle protein and vascular endothelial growth factors (ClinicalTrials.Gov, identifier \# NCT00882180). Thus the translation potential of RNAi mediated blockade of HCC is largely unexplored. Targeting tumor-specific GAPDH could be achieved either by targeted-delivery or selective-activation of si/shRNA in the tumor. The targeted delivery is within the limits of loco-regional therapies whereas selective activation involves a strategy of molecular cloning. For instance, if the expression of GAPDH-shRNA is regulated by the known marker of HCC (the alpha-fetoprotein, AFP) it would enable us to selectively target AFP-positive HCC. In such a scenario, even if the GAPDH-shRNA inadvertently enters into normal, non-cancerous cells, the toxicity will be prevented as the silencing effect is under the control of the presence of HCC marker, AFP.

\section{FUTURE DIRECTIONS}

Successful translation of any potential chemotherapeutic for cancer essentially relies on the molecular-specificity of the therapeutic agent and its selectivity in targeting tumor. However, for liver cancer such as $\mathrm{HCC}$, there is an additional requirement that needs to be fulfilled for successful practice in the clinics; that is the potential new drug candidates must exhibit an extremely favorable toxicity profile in order to preserve the functionally normal liver. This necessity is due to the fact that liver cancer typically arises in the context of an underlying liver disease (e.g. cirrhosis) making the liver especially susceptible to any kind of therapeutic insult. Thus ideal molecular targets will be those that are critically required for cancer cell proliferation, chemoresistance and metastasis. Since GAPDH is known to be involved in all of these processes (Figure 5) it is an extremely attractive 
target for therapy.

Identifying a specific inhibitor can be as difficult as identifying the molecular-target. Based on our current understanding of GAPDH and its functions, it is evident that inhibition of either the glycolytic role or interference with its non-glycolytic actions is sufficient to block GAPDH. Akin to this, several motifs have been predicted in the peptide sequence of GAPDH that can be attributed to its multiple functions (Figure 6). Preclinical evaluation of GAPDH inhibitors to date has been shown to inhibit its glycolytic role. Whether such inhibitors affect the PTMs, and their anticancer effects are PTM-inhibition dependent, have yet to be proven. It certainly appears that PTM remains an area that requires intense research especially since the majority of the non-glycolytic functions of GAPDH are affected by PTMs. For example it is known that phosphorylation of the Thr 237 decreases the nuclear translocation of GAPDH and blocks its apoptotic role [82]. Presumably, screening and developing inhibitors that would inhibit Thr 237 phosphorylation could restore GAPDH's-apoptotic role. Likewise, Akt2 kinase has been documented to mediate phosphorylation of $\mathrm{Thr}$ 237. Hence inhibitors specific for Akt2 kinase could support GAPDH dependent apoptosis. Interestingly, another PTM, the O-GlcNAcylation of GAPDH at Thr (227) has been shown to allow its nuclear translocation to facilitate its non-glycolytic functions [83] suggesting O-GlcNAcylation as a possible target. However, the translational potential of such an anti-GAPDH approach lies in the successful treatment of HCC in clinically relevant experimental models. Hence, further research is necessary in order to evaluate (a) the feasibility of delivery of GAPDH-shRNA under Ultra-sound guidance to target GAPDH in an orthotopic liver tumor model and (b) the molecular specificity of AFP-dependent silencing of GAPDH in HCC.

Recently, gene (silencing) therapy and / or antibody based therapeutics have been shown to be very effective in loco-regional therapies however, their selectivity in targeting tumor cells and sparing normal hepatocytes would require tumor cell-specific functional activation of such therapeutics. This is plausible, if the functional activation of such therapeutics is dependent on the presence of a tumor-specific protein (e.g. AFP in some cases of HCC). Preclinical studies in line with this principle of tumor-specific functional activation have been documented and further studies are required to evaluate this selective silencing of GAPDH in HCC in relevant models mimicking the clinical set-up (e.g. spontaneous HCC, orthotopic liver tumor, image-guided therapy etc).

The failure of majority of the antimetabolites during clinical translation may be attributed to the plasticity of tumor cells where "alternative or complementary feeder pathways" can substitute for the disrupted metabolism. In other words, the complex network of tumor metabolism demonstrates innate flexibility to overcome or withstand disruption of any single biochemical pathway or inhibition of any particular molecular target. Hence, concurrent inhibition of complementary metabolic pathways (e.g., glycolysis, PPP, cellular redox balance) might prove to be a vital therapeutic strategy.

In summary, "targeting early steps of glucose metabolism (e.g. GAPDH activity) will enable us to block intracellular energy production and other critical pathways linked to it (e.g. macromolecular biosynthesis), eventually causing cancer cell death". Future research focusing on the targeted (via loco-regional therapy) and selective (e.g. 3-BrPA) inhibition of GAPDH would provide us an opportunity to acquire better armamentarium in treating HCC.

\section{ACKNOWLEDGEMENTS}

We gratefully acknowledge the support by the Charles Wallace Pratt Research Fund and Abdulrahman Abdulmalik Research Fund. Authors declare that there is no conflict of interests.

\section{REFERENCE}

1. Hopkins AL, Groom CR. The druggable genome. Nat Rev Drug Discov. 2002; 1: 727-730.

2. Sirover MA. New nuclear functions of the glycolytic protein, glyceraldehyde-3-phosphate dehydrogenase, in mammalian cells. J Cell Biochem. 2005; 95: 45-52.

3. Sirover MA. On the functional diversity of glyceraldehyde3-phosphate dehydrogenase: Biochemical mechanisms and regulatory control. Biochim Biophys Acta. 2011; 1810: 741-751.

4. Sirover MA. Subcellular dynamics of multifunctional protein regulation: Mechanisms of GAPDH intracellular translocation. J Cell Biochem. 2012; 113: 2193-2200.

5. Colell A, Ricci JE, Tait S, Milasta S, Maurer U, BouchierHayes L, Fitzgerald P, Guio-Carrion A, Waterhouse NJ, Li CW, Mari B, Barbry P, Newmeyer DD, Beere HM, Green DR. GAPDH and autophagy preserve survival after apoptotic cytochrome c release in the absence of caspase activation. Cell. 2007; 129: 983-997.

6. Colell A, Green DR, Ricci JE. Novel roles for GAPDH in cell death and carcinogenesis. Cell Death Differ. 2009; 16: 1573-1581.

7. El-Serag HB. Hepatocellular carcinoma. N Engl J Med. 2011; 365: 1118-1127.

8. Llovet JM, Bustamante J, Castells A, Vilana R, Ayuso Mdel C, Sala M, Bru C, Rodes J, Bruix J. Natural history of untreated nonsurgical hepatocellular carcinoma: Rationale for the design and evaluation of therapeutic trials. Hepatology. 1999; 29: 62-67.

9. Lencioni R. Loco-regional treatment of hepatocellular carcinoma. Hepatology. 2010; 52: 762-773. 
10. Liapi E, Geschwind JF. Transcatheter and ablative therapeutic approaches for solid malignancies. J Clin Oncol. 2007; 25: 978-986.

11. Liapi E, Geschwind JF. Intra-arterial therapies for hepatocellular carcinoma: Where do we stand? Ann Surg Oncol. 2010; 17: 1234-1246.

12. Ahmed M, Brace CL, Lee FT,Jr, Goldberg SN. Principles of and advances in percutaneous ablation. Radiology. 2011; 258: 351-369.

13. Naitoh Y, Kawauchi A, Mizutani Y, Ukimura O, Fujito A, Nakao M, Kojima Y, Nonomura N, Okuyama A, Miki T. Significant antitumor effect of intratumoral ethanol injection on renal cell carcinoma. Eur Urol. 2003; 44: 452457.

14. Yoon DJ, Kwan BH, Chao FC, Nicolaides TP, Phillips JJ, Lam GY, Mason AB, Weiss WA, Kamei DT. Intratumoral therapy of glioblastoma multiforme using genetically engineered transferrin for drug delivery. Cancer Res. 2010; 70: 4520-4527.

15. Veltri A, Moretto P, Doriguzzi A, Pagano E, Carrara G, Gandini G. Radiofrequency thermal ablation (RFA) after transarterial chemoembolization (TACE) as a combined therapy for unresectable non-early hepatocellular carcinoma (HCC). Eur Radiol. 2006; 16: 661-669.

16. Vucur M, Roderburg C, Bettermann K, Tacke F, Heikenwalder M, Trautwein C, Luedde T. Mouse models of hepatocarcinogenesis: What can we learn for the prevention of human hepatocellular carcinoma? Oncotarget. 2010; 1: 373-378.

17. Cervello M, McCubrey JA, Cusimano A, Lampiasi N, Azzolina A, Montalto G. Targeted therapy for hepatocellular carcinoma: Novel agents on the horizon. Oncotarget. 2012; 3: 236-260.

18. Yun J, Rago C, Cheong I, Pagliarini R, Angenendt P, Rajagopalan H, Schmidt K, Willson JK, Markowitz S, Zhou S, Diaz LA,Jr, Velculescu VE, Lengauer C, Kinzler KW, Vogelstein B, Papadopoulos N. Glucose deprivation contributes to the development of KRAS pathway mutations in tumor cells. Science. 2009; 325: 1555-1559.

19. Madan E, Gogna R, Bhatt M, Pati U. Regulation of glucose metabolism by p53: Emerging new roles for the tumor suppressor. 2011; 2: 948-957.

20. Kroemer G, Pouyssegur J. Tumor cell metabolism: Cancer's achilles' heel. Cancer Cell. 2008; 13: 472-482.

21. Wolf A, Agnihotri S, Guha A. Targeting metabolic remodeling in glioblastoma multiforme. Oncotarget. 2010; 1: $552-562$.

22. Kuo MT, Savaraj N, Feun LG. Targeted cellular metabolism for cancer chemotherapy with recombinant argininedegrading enzymes. Oncotarget. 2010; 1: 246-251.

23. Hugo-Wissemann D, Anundi I, Lauchart W, Viebahn $\mathrm{R}$, de Groot H. Differences in glycolytic capacity and hypoxia tolerance between hepatoma cells and hepatocytes. Hepatology. 1991; 13: 297-303.
24. Kitamura K, Hatano E, Higashi T, Narita M, Seo S, Nakamoto Y, Yamanaka K, Nagata H, Taura K, Yasuchika $\mathrm{K}$, Nitta T, Uemoto S. Proliferative activity in hepatocellular carcinoma is closely correlated with glucose metabolism but not angiogenesis. J Hepatol. 2011; 55: 846-857.

25. Fritz V, Fajas L. Metabolism and proliferation share common regulatory pathways in cancer cells. Oncogene. 2010; 29: 4369-4377.

26. Dang CV, Hamaker M, Sun P, Le A, Gao P. Therapeutic targeting of cancer cell metabolism. J Mol Med. 2011; 89: 205-212.

27. Geschwind JF, Georgiades CS, Ko YH, Pedersen PL. Recently elucidated energy catabolism pathways provide opportunities for novel treatments in hepatocellular carcinoma. Expert Rev Anticancer Ther. 2004; 4: 449-457.

28. Zang WQ, Fieno AM, Grant RA, Yen TS. Identification of glyceraldehyde-3-phosphate dehydrogenase as a cellular protein that binds to the hepatitis B virus posttranscriptional regulatory element. Virology. 1998; 248: 46-52.

29. Li Y, Huang T, Zhang X, Wan T, Hu J, Huang A, Tang H. Role of glyceraldehyde-3-phosphate dehydrogenase binding to hepatitis B virus posttranscriptional regulatory element in regulating expression of HBV surface antigen. Arch Virol. 2009; 154: 519-524.

30. Petrik J, Parker H, Alexander GJ. Human hepatic glyceraldehyde-3-phosphate dehydrogenase binds to the poly(U) tract of the 3' non-coding region of hepatitis $\mathrm{C}$ virus genomic RNA. J Gen Virol. 1999; 80 ( Pt 12): 31093113.

31. De BP, Gupta S, Zhao H, Drazba JA, Banerjee AK. Specific interaction in vitro and in vivo of glyceraldehyde3-phosphate dehydrogenase and LA protein with cis-acting RNAs of human parainfluenza virus type 3. J Biol Chem. 1996; 271: 24728-24735.

32. Yang SH, Liu ML, Tien CF, Chou SJ, Chang RY. Glyceraldehyde-3-phosphate dehydrogenase (GAPDH) interaction with 3' ends of japanese encephalitis virus RNA and colocalization with the viral NS5 protein. J Biomed Sci. 2009; 16 : 40.

33. Yamamoto S, Takashima S, Ogawa H, Kuroda $\mathrm{T}$, Yamamoto M, Takeda A, Nakmaura H. Granulocytecolony-stimulating-factor-producing hepatocellular carcinoma. J Gastroenterol. 1999; 34: 640-644.

34. Araki K, Kishihara F, Takahashi K, Matsumata T, Shimura T, Suehiro T, Kuwano H. Hepatocellular carcinoma producing a granulocyte colony-stimulating factor: Report of a resected case with a literature review. Liver Int. 2007; 27: 716-721.

35. Zhu XD, Zhang JB, Zhuang PY, Zhu HG, Zhang W, Xiong YQ, Wu WZ, Wang L, Tang ZY, Sun HC. High expression of macrophage colony-stimulating factor in peritumoral liver tissue is associated with poor survival after curative resection of hepatocellular carcinoma. J Clin Oncol. 2008; 26: $2707-2716$. 
36. Zhou Y, Yi X, Stoffer JB, Bonafe N, Gilmore-Hebert M, McAlpine J, Chambers SK. The multifunctional protein glyceraldehyde-3-phosphate dehydrogenase is both regulated and controls colony-stimulating factor-1 messenger RNA stability in ovarian cancer. Mol Cancer Res. 2008; 6: 1375-1384.

37. Eferl R, Ricci R, Kenner L, Zenz R, David JP, Rath M, Wagner EF. Liver tumor development. c-jun antagonizes the proapoptotic activity of p53. Cell. 2003; 112: 181-192.

38. Demarse NA, Ponnusamy S, Spicer EK, Apohan E, Baatz JE, Ogretmen B, Davies C. Direct binding of glyceraldehyde 3-phosphate dehydrogenase to telomeric DNA protects telomeres against chemotherapy-induced rapid degradation. J Mol Biol. 2009; 394: 789-803.

39. Gong Y, Cui L, Minuk GY. Comparison of glyceraldehyde3-phosphate dehydrogenase and 28s-ribosomal RNA gene expression in human hepatocellular carcinoma. Hepatology. 1996; 23: 734-737.

40. Lee MN, Ha SH, Kim J, Koh A, Lee CS, Kim JH, Jeon H, Kim DH, Suh PG, Ryu SH. Glycolytic flux signals to mTOR through glyceraldehyde-3-phosphate dehydrogenase-mediated regulation of rheb. Mol Cell Biol. 2009; 29: 3991-4001.

41. Villanueva A, Chiang DY, Newell P, Peix J, Thung S, Alsinet C, Tovar V, Roayaie S, Minguez B, Sole M, Battiston C, Van Laarhoven S, Fiel MI, Di Feo A, Hoshida Y, Yea S, Toffanin S, Ramos A, Martignetti JA, Mazzaferro V, Bruix J, Waxman S, Schwartz M, Meyerson M, Friedman SL, Llovet JM. Pivotal role of mTOR signaling in hepatocellular carcinoma. Gastroenterology. 2008; 135: 1972-83, 1983.e1-11.

42. Ho C, Wang C, Mattu S, Destefanis G, Ladu S, Delogu S, Armbruster J, Fan L, Lee SA, Jiang L, Dombrowski F, Evert M, Chen X, Calvisi DF. AKT (v-akt murine thymoma viral oncogene homolog 1) and N-ras (neuroblastoma ras viral oncogene homolog) coactivation in the mouse liver promotes rapid carcinogenesis by way of mTOR (mammalian target of rapamycin complex 1), FOXM1 (forkhead box M1)/SKP2, and c-myc pathways. Hepatology. 2012; 55: 833-845.

43. Piguet AC, Semela D, Keogh A, Wilkens L, Stroka D, Stoupis C, St-Pierre MV, Dufour JF. Inhibition of mTOR in combination with doxorubicin in an experimental model of hepatocellular carcinoma. J Hepatol. 2008; 49: 78-87.

44. Tam KH, FAU - Yang ZF, Yang ZF, FAU - Lau CK, Lau CK, FAU - Lam CT, Lam CT, FAU - Pang RWC, Pang RW, FAU - Poon RTP, Poon RT. Inhibition of mTOR enhances chemosensitivity in hepatocellular carcinoma. Cancer Lett.2009 Jan 18;273(2):201-9.Epub 2008 Sep 27.

45. Chinnakotla S, Davis GL, Vasani S, Kim P, Tomiyama K, Sanchez E, Onaca N, Goldstein R, Levy M, Klintmalm GB. Impact of sirolimus on the recurrence of hepatocellular carcinoma after liver transplantation. Liver Transpl. 2009; 15: 1834-1842.

46. Markman B, Dienstmann R, Tabernero J. Targeting the
PI3K/Akt/mTOR pathway--beyond rapalogs. Oncotarget. 2010; 1: 530-543.

47. Chappell WH, Steelman LS, Long JM, Kempf RC, Abrams SL, Franklin RA, Basecke J, Stivala F, Donia M, Fagone P, Malaponte G, Mazzarino MC, Nicoletti F, Libra M, Maksimovic-Ivanic D, Mijatovic S, Montalto G, Cervello M, Laidler P, Milella M, Tafuri A, Bonati A, Evangelisti C, Cocco L, Martelli AM, McCubrey JA. Ras/Raf/MEK/ ERK and PI3K/PTEN/Akt/mTOR inhibitors: Rationale and importance to inhibiting these pathways in human health. Oncotarget. 2011; 2: 135-164.

48. Kudo M. mTOR inhibitor for the treatment of hepatocellular carcinoma. Dig Dis. 2011; 29: 310-315.

49. Yang Z, Zhang L, Ma A, Liu L, Li J, Gu J, Liu Y. Transient mTOR inhibition facilitates continuous growth of liver tumors by modulating the maintenance of CD133+ cell populations. PLoS One. 2011; 6: e28405.

50. Tarze A, Deniaud A, Le Bras M, Maillier E, Molle D, Larochette N, Zamzami N, Jan G, Kroemer G, Brenner C. GAPDH, a novel regulator of the pro-apoptotic mitochondrial membrane permeabilization. Oncogene. 2007; 26: 2606-2620.

51. Sundararaj KP, Wood RE, Ponnusamy S, Salas AM, Szulc Z, Bielawska A, Obeid LM, Hannun YA, Ogretmen B. Rapid shortening of telomere length in response to ceramide involves the inhibition of telomere binding activity of nuclear glyceraldehyde-3-phosphate dehydrogenase. J Biol Chem. 2004; 279: 6152-6162.

52. Tristan C, Shahani N, Sedlak TW, Sawa A. The diverse functions of GAPDH: Views from different subcellular compartments. Cell Signal. 2011; 23: 317-323.

53. Fiume L, Manerba M, Vettraino M, Di Stefano G. Impairment of aerobic glycolysis by inhibitors of lactic dehydrogenase hinders the growth of human hepatocellular carcinoma cell lines. Pharmacology. 2010; 86: 157-162.

54. Ahn KJ, Hwang HS, Park JH, Bang SH, Kang WJ, Yun M, Lee JD. Evaluation of the role of hexokinase type II in cellular proliferation and apoptosis using human hepatocellular carcinoma cell lines. J Nucl Med. 2009; 50: 1525-1532.

55. Hulleman E, Kazemier KM, Holleman A, VanderWeele DJ, Rudin CM, Broekhuis MJ, Evans WE, Pieters R, Den Boer ML. Inhibition of glycolysis modulates prednisolone resistance in acute lymphoblastic leukemia cells. Blood. 2009; 113: 2014-2021.

56. Epner DE, Partin AW, Schalken JA, Isaacs JT, Coffey DS. Association of glyceraldehyde-3-phosphate dehydrogenase expression with cell motility and metastatic potential of rat prostatic adenocarcinoma. Cancer Res. 1993; 53: 19951997.

57. Phadke MS, Krynetskaia NF, Mishra AK, Krynetskiy E. Glyceraldehyde 3-phosphate dehydrogenase depletion induces cell cycle arrest and resistance to antimetabolites in human carcinoma cell lines. J Pharmacol Exp Ther. 2009; 
331: 77-86.

58. Xing C, LaPorte JR, Barbay JK, Myers AG. Identification of GAPDH as a protein target of the saframycin antiproliferative agents. Proc Natl Acad Sci U S A. 2004; 101: 5862-5866.

59. Endo A, Hasumi K, Sakai K, Kanbe T. Specific inhibition of glyceraldehyde-3-phosphate dehydrogenase by koningic acid (heptelidic acid). J Antibiot (Tokyo). 1985; 38: 920925.

60. Kato M, Sakai K, Endo A. Koningic acid (heptelidic acid) inhibition of glyceraldehyde-3-phosphate dehydrogenases from various sources. Biochim Biophys Acta. 1992; 1120: 113-116.

61. Kumagai S, Narasaki R, Hasumi K. Glucose-dependent active ATP depletion by koningic acid kills high-glycolytic cells. Biochem Biophys Res Commun. 2008; 365: 362-368.

62. Ganapathy-Kanniappan S, Geschwind JF, Kunjithapatham R, Buijs M, Vossen JA, Tchernyshyov I, Cole RN, Syed LH, Rao PP, Ota S, Vali M. Glyceraldehyde-3phosphate dehydrogenase (GAPDH) is pyruvylated during 3-bromopyruvate mediated cancer cell death. Anticancer Res. 2009; 29: 4909-4918.

63. Pereira da Silva AP, El-Bacha T, Kyaw N, dos Santos RS, da-Silva WS, Almeida FC, Da Poian AT, Galina A. Inhibition of energy-producing pathways of HepG2 cells by 3-bromopyruvate. Biochem J. 2009; 417: 717-726.

64. Ganapathy-Kanniappan S, Vali M, Kunjithapatham R, Buijs M, Syed LH, Rao PP, Ota S, Kwak BK, Loffroy R, Geschwind JF. 3-bromopyruvate: A new targeted antiglycolytic agent and a promise for cancer therapy. Curr Pharm Biotechnol. 2010; 11: 510-517.

65. McKee RW, Wong W, Landman M. Effects of iodoacetate on glycolysis and respiration in ehrlich-lettre ascites carcinoma cells. Biochim Biophys Acta. 1965; 105: 410423.

66. Sanchez-Arago M, Cuezva JM. The bioenergetic signature of isogenic colon cancer cells predicts the cell death response to treatment with 3-bromopyruvate, iodoacetate or 5-fluorouracil. J Transl Med. 2011; 9: 19.

67. Lee HJ, Howell SK, Sanford RJ, Beisswenger PJ. Methylglyoxal can modify GAPDH activity and structure. Ann N Y Acad Sci. 2005; 1043: 135-145.

68. Ray M, Basu N, Ray S. Inactivation of glyceraldehyde3-phosphate dehydrogenase of human malignant cells by methylglyoxal. Mol Cell Biochem. 1997; 177: 21-26.

69. Halder J, Ray M, Ray S. Inhibition of glycolysis and mitochondrial respiration of ehrlich ascites carcinoma cells by methylglyoxal. Int J Cancer. 1993; 54: 443-449.

70. Kim JW, Kim TE, Kim YK, Kim YW, Kim SJ, Lee JM, Kim IK, Namkoong SE. Antisense oligodeoxynucleotide of glyceraldehyde-3-phosphate dehydrogenase gene inhibits cell proliferation and induces apoptosis in human cervical carcinoma cell lines. Antisense Nucleic Acid Drug Dev. 1999; 9: 507-513.
71. Ganapathy-Kanniappan S, Kunjithapatham R, Torbenson MS, Rao PP, Carson KA, Buijs M, Vali M, Geschwind JF. Human hepatocellular carcinoma in a mouse model: Assessment of tumor response to percutaneous ablation by using glyceraldehyde-3-phosphate dehydrogenase antagonists. Radiology. 2012; 262: 834-845.

72. Hong $\mathrm{K}$, Geschwind JF. Locoregional intra-arterial therapies for unresectable intrahepatic cholangiocarcinoma. Semin Oncol. 2010; 37: 110-117.

73. Liapi E, Geschwind JF. Interventional oncology: New options for interstitial treatments and intravascular approaches: Targeting tumor metabolism via a locoregional approach: A new therapy against liver cancer. J Hepatobiliary Pancreat Sci. 2010; 17: 405-406.

74. Xu Y, Chenna V, Hu C, Sun HX, Khan M, Bai H, Yang XR, Zhu QF, Sun YF, Maitra A, Fan J, Anders RA. A polymeric nanoparticle encapsulated hedgehog pathway inhibitor HPI-1 (NanoHHI) inhibits systemic metastases in an orthotopic model of human hepatocellular carcinoma. Clin Cancer Res. 2011 Aug 25. [Epub ahead of print].

75. Zhou Q, Ching AK, Leung WK, Szeto CY, Ho SM, Chan PK, Yuan YF, Lai PB, Yeo W, Wong N. Novel therapeutic potential in targeting microtubules by nanoparticle albuminbound paclitaxel in hepatocellular carcinoma. Int J Oncol. 2011; 38: 721-731.

76. Hong K, Georgiades CS, Geschwind JF. Technology insight: Image-guided therapies for hepatocellular carcinoma--intra-arterial and ablative techniques. Nat Clin Pract Oncol. 2006; 3: 315-324.

77. Ko YH, Pedersen PL, Geschwind JF. Glucose catabolism in the rabbit VX2 tumor model for liver cancer: Characterization and targeting hexokinase. Cancer Lett. 2001; 173: 83-91.

78. Geschwind JF, Ko YH, Torbenson MS, Magee C, Pedersen PL. Novel therapy for liver cancer: Direct intraarterial injection of a potent inhibitor of ATP production. Cancer Res. 2002; 62: 3909-3913.

79. Ko YH, Smith BL, Wang Y, Pomper MG, Rini DA, Torbenson MS, Hullihen J, Pedersen PL. Advanced cancers: Eradication in all cases using 3-bromopyruvate therapy to deplete ATP. Biochem Biophys Res Commun. 2004; 324 : 269-275.

80. Pawlik TM, Reyes DK, Cosgrove D, Kamel IR, Bhagat N, Geschwind JF. Phase II trial of sorafenib combined with concurrent transarterial chemoembolization with drugeluting beads for hepatocellular carcinoma. J Clin Oncol. 2011; .

81. Xu C, Lee SA, Chen X. RNA interference as therapeutics for hepatocellular carcinoma. Recent Pat Anticancer Drug Discov. 2011; 6: 106-115.

82. Huang Q, Lan F, Zheng Z, Xie F, Han J, Dong L, Xie Y, Zheng F. Akt2 kinase suppresses glyceraldehyde-3phosphate dehydrogenase (GAPDH)-mediated apoptosis in ovarian cancer cells via phosphorylating GAPDH at 
threonine 237 and decreasing its nuclear translocation. J Biol Chem. 2011; 286: 42211-42220.

83. Park J, Han D, Kim K, Kang Y, Kim Y. O-GlcNAcylation disrupts glyceraldehyde-3-phosphate dehydrogenase homotetramer formation and mediates its nuclear translocation. Biochim Biophys Acta. 2009; 1794: 254-262. 